\title{
Management of Multiple Pregnancy
}

\section{Joachim W Dudenhausen}

Klinik für Geburtsmedizin, Charité - Universitätsmedizin Berlin, Berlin, Germany

Correspondence: Joachim W Dudenhausen, Klinik für Geburtsmedizin, Charité - Universitätsmedizin Berlin, Campus VirchowKlinikum, Augustenburger Platz 1, D-13353, Berlin, Germany, e-mail: Joachim.Dudenhausen@charite.de

\begin{abstract}
Background: Changing delivery age and successes in reproductive have medicine induced an increase in incidence of the multiples in the industrialized world.

Methods: Selective literature research was performed complemented by the autor's clinical experience and national and international guidelines.

Results: The risks of prematurity, IUGR, and antenatal demise are raised. Maternal risks are pre-eclampsia, diabetes, and bleeding during delivery. Prenatal and genetic diagnostics including ultrasonography are the most important methods of supervision during pregnancy. These are significant for diagnosis of twin-to-twin transfusion syndrome and cygosity.

Consequences: Cooperation betweeen prenatal medicine, obstetrics and neonatology is important for the care of mothers with multiples.

Keywords: Multiples, prematurity, IUGR, ultrasound.
\end{abstract}

\section{FREQUENCY}

The frequency at which multiple gestations occur varies considerably from country-to-country. The rule established in 1895 by Hellin to assess the frequency of multiple pregnancies essentially continues in force even today: if twins are born at a frequency of 1:85, triplets will be born at a frequency of 1:85 $\times 85$ and quadruplets at a rate of 1:85 $\times 85 \times 85$. These rates are considerably higher in early pregnancy. Boklage followed 325 twin pregnancies, of which $19 \%$ ended on the due date with twins being born, $39 \%$ were, singleton births, and in $43 \%$ of the cases, no live child was born. He calculated the likely conception rate for twins as being $1: 8 .^{7}$

In most European countries, the rate of twin births decreased in the 1960s from about 12 per 1,000 pregnancies to around 9.5, subsequently increasing again from the early 1980s onwards to about 12 per 1,000 pregnancies, and reaching 13 to 14 twin births per 1,000 pregnancies around 1990. The development apparent in the course of the 1960s and 1970s was primarily caused by changes in the age structure of pregnant women (initially, the numbers of younger pregnant women increased, later followed by an increase of pregnant women over the age of 35). The increased rate of multiple pregnancies following 1990 is generally perceived to be a result of the efforts of reproductive medicine.
The increased frequency of high-order multiple pregnancies since the late 1980s can only be termed dramatic. In the old federal States of the Federal Republic of Germany, the rate of triplets increased between 1975 and 1990 by about $170 \%$; in the Netherlands, it did so at a rate of $300 \%$. Generally, induced ovulation and IVF are regarded to be the causes at the root of this development.

Where, the frequency of dizygotic twins is concerned, the occurrence of multiple pregnancies in the mother's family has considerably greater impact than the instance of multiples in the father's family does. About $2 \%$ of the women who themselves were a dizygotic twin gave birth to twins. By contrast, the frequency of twins being born by women whose husbands were dizygotic twins was only around $1 \% .^{5,6}$

\section{ADAPTION OF THE MOTHERS TO THEIR MULTIPLE PREGNANCIES}

As a general rule, the mothers' organisms will undergo greater physiological changes in multiple pregnancies than in singleton pregnancies. Thus, the circulating blood volume will increase by 25 to $30 \%$ at its peak in the 32nd through 36th week of pregnancy, whereas, this increase will amount to 50 to $60 \%$ in a twin pregnancy. In other words, a twin pregnancy will entail an additional volume of $500 \mathrm{ml}$ of blood over the increased blood volume occurring in a 
singleton pregnancy. Accordingly, a pregnant woman carrying multiples who is suffering from heart disease will be subject to extreme strain in the 32nd through 36th week of pregnancy.

The increase in the amount of blood and the greater need for iron and folic acid engendered by the growing fetuses predispose pregnant women to anemia, a condition frequently encountered during any pregnancy.

The larger dimensions of the uterus carrying multiples will engender mechanical disruptions to the function of the visceral organs, while the elevation of the diaphragm will impair lung function.

\section{DURATION OF THE PREGNANCY}

On average, multiple pregnancies are considerably shorter. In the United States, the preterm birth rate in, 1987 was $44.5 \%$ for twins (pregnancy week $<37+0$ ) as opposed to $9.4 \%$ for singletons. The average duration of twin pregnancies was the 36th week of pregnancy, as opposed to week 39 for singleton births.

The average gestational age of triplets at their birth was 32 weeks, while quadruplets reached a gestational age of 30 weeks.

The causes for this reduced pregnancy duration were seen to be given in the mechanical strain on the cervix, as well as the relatively reduced blood flow through the uterus and the relatively reduced placenta function when seen against the weight of the fetuses and the placenta. Moreover, the formation of gap junctions as the result of the high estrogen activity and of prostaglandin synthesis, coupled with the relative reduction of the progesterone activity in multiple pregnancies, seem to likewise contribute to reducing pregnancy duration.

\section{ULTRASOUND DIAGNOSIS}

The higher rate at which multiple pregnancies are detected has served to decrease perinatal mortality. The ultrasound examinations performed in accordance with the guidelines issued by the German federal committee of physicians and health insurance funds on proper medical care during pregnancy and postpartum have caused prenatal detection rates of multiple gestations close to $100 \%$. Such an early diagnosis has an important role to play in managing the pregnancy as well as in supervising the mother and her children, while also defining the intrapartal processes and contributing to an optimal preparation of the parents.
It is possible to diagnose a multiple pregnancy, determine the gestational age and monitor fetal growth in accordance with the norm curves. At this juncture, it is important to note that fetal growth curves statistically do not differ for singletons and twins where, fetal head diameter and femur length are concerned. This means that differentiated examinations to detect any malformations must be performed.

Moreover, as examinations specific to multiple pregnancies, it is important to determine zygocity and placentation. ${ }^{13,15}$

\section{BASES OF ZYGOCITY DETERMINATION}

Monozygotic twins are formed when an embryo splits into two different cells. Of 1,000 births, approximately four will be monozygotic twins. Embryofetal mortality is higher for monozygotic twins than it is for dizygotic twins and singletons. The rate of serious malformations is cited as $2.3 \%$, as opposed to $1 \%$ for singletons; for lesser malformations, it is $4.1 \%$ as opposed to $2.5 \%$. Statistically, monochorionic, monoamniotic twins are subject to a greater frequency of such malformations, in which context twin girls represent the less serious of these cases. ${ }^{1}$ If the embryo splits in the period up to the fifth day following fertilization, dichorionic, diamniotic twins will develop (around 30\%). If the embryo splits between the fifth and seventh day following fertilization, monochorionic, diamniotic twins will form (around $70 \%$ ). If the split occurs in the period following day 8, monochorionic, monoamniotic twins will develop (around 1\%). Conjoined twins are engendered by an incomplete split in the period from day 15 until day 17 following fertilization; in Europe they occur at a rate of 1 in 33,000 births.

Dizygotic twins are created by the fertilization of two different egg cells from two different follicles. The growth of the follicles is regulated by gonadotropins. The thesis has been brought forward that higher levels of follicle stimulating hormones are associated with a higher rate of dizygotic twins. FSH production is influenced by periods of light and darkness; thus, it is said that in Scandinavia, the rate of dizygotic twins is higher for pregnancies conceived in July while it is lower for conceptions in January. The likelihood of conceiving dizygotic twins increases with the mother's age until the age of 39, and then decreases again; it also decreases during periods in which the population suffers from malnutrition. 
High-order multiples may result from the fertilization of one, two or several egg cells, or they may be engendered by the split of one or several fertilized egg cells, so that a mixture of a dizygotic and monozygotic multiple pregnancy may occur.

Awareness of the zygocity is an important pre-requisite for correctly assessing the risk factors that may arise in the course of the pregnancy. For example, should one of the twins experience an intrauterine maldevelopment, this may result in different growth rates for the fetuses, as may also be the case in the context of the twin transfusion syndrome that monozygotic twins may suffer from. Today, ultrasound examinations have become indispensable in determining zygocity. In dichorial pregnancies, a lambda-shaped structure where, the fetal membranes join the placenta will be visible in the course of the 10th until the 15th week of pregnancy. Major diagnostic findings in such pregnancies include separate placentas or a (fused) placenta, membrane thickness (monozygotic twins will have a thin membrane, while dizygotic twins will have a thick one).

The physiological process in which an embryo, or the early fetuses of a multiple pregnancy, disappear (so-called "vanishing twin") will lead to their resorption, an empty amniotic sac, or a fetus papyraceus. In clinical terms, this process will generally only be indicated by bleeding ex utero.

\section{MONITORING FETAL WELL-BEING}

Determining the biophysical profile of the multiples by establishing the volume of the fluid in their amniotic sac, performing a nonstress test, checking fetal breathing movements and other movements is an essential aspect of monitoring their well-being during pregnancy.

Doppler blood flow assessments are important particularly whenever, risks are determined (weight difference, TTS and such like) and must govern any clinical examination.

\section{PRENATAL GENETIC DIAGNOSTICS}

Since the 1970s, pregnant women have had the opportunity to have a genetic diagnosis performed; this naturally also applies to multiple pregnancies.

Basically, amniocentesis in the 2nd trimester, CVS or early amniocentesis are the diagnostic methods of choice. The rate of complications for amniocentesis performed in multiple pregnancies is cited as being five times as high as that given for singleton pregnancies. ${ }^{2}$

\section{SELECTIVE FETOCIDE}

The gravest risks most frequently encountered in multiple pregnancies are the reduced pregnancy duration and the increased risks for the mothers. For triplets, they are as follows: $20 \%$ pre-eclampsia, 30\% anemia, 35\% postpartum bleeding; for quadruplets, the risk rates are: $32 \%$ preeclampsia, 25\% anemia, 21\% postpartum bleeding.

Based on experience with cases in which a fetocide is indicated due to the malformation of one of the multiples, combined with the intention to reduce the above risks, various methods were used to selectively reduce pregnancies of high-order multiples to a twin pregnancy ${ }^{3}$ (hysterotomy, heart punction, injection of air or of cardio-toxic substances). Groups experienced in this work recommend the transabdominal, intrathoracal injection of calium chloride around the 11th to 12th week; in the view held by many authors, the benefit to the remaining offspring of the multiple gestation justifies this procedure. Prior to making such an injection, it should be considered that where this is done to monozygotic twins, the cardiotoxic substances injected into one twin will transfuse to the other twin and thus place the latter at considerable risk. Pregnancy loss is to be expected in $10 \%$ of the cases of such selective fetocide. ${ }^{8}$

In ethical terms, selective fetocide is highly problematic and should be avoided by taking appropriate measures available to reproductive medicine.

\section{TWIN-TWIN TRANSFUSION SYNDROME (TTTS)}

Monozygotic, monochorial twin pregnancies will have interfetal vascular connections at the level of the placenta, as well as arterio-arterial and veno-venous anastomoses on the chorion plate and arteriovenous shunts in the cotyledons. These are the basis for a redistribution of the blood among the fetuses, the causes of which have not yet been conclusively established. Possibly, a higher vascular resistance in the placental circulation of the donor as a result of a placental dysfunction may be the cause of such blood redistribution. The result is that one of the twins will grow larger, become polyglobule (recipient twin) and will have excessive amniotic fluid (polyhydramnios), while the donor twin will experience growth retardation, become anemic and will have too little amniotic fluid (oligohydramnios).

All diagnostic efforts should be informed by the relation of intrauterine weight differences (of more than 20\%) to the different volume of amniotic fluid that can be established in ultrasound examinations (polyhydramnios for the recipient 
twin, oligohydramnios for the donor twin).$^{18}$ The volume of amniotic fluid may decrease to such a degree that the donor twin, as the smaller one, is pinned to the side of the gestational sac ("stuck twin"). ${ }^{4}$

The overall mortality rates for TTTS are very high (56 to $100 \%)$. In 3 to $5 \%$ of the cases, fetal death occurs already in utero. Following the death of a twin, twin embolization syndrome occurs in up to $14 \%$ of the cases. This term refers to arterial hypotony and the dead fetus transfusing thromboplastic material into the surviving fetus. The consequences are a diffuse intravascular coagulation and/ or cardiac infarctions accompanied by serious neurological collapses, so that an intervention should absolutely be made prior to the intrauterine death of the fetus. Today, TTS is treated using the following therapies:

1. Repeated amniocentesis and amnioreduction; while the pathogenetic mechanism of this treatment is unclear, repeated amniocentesis has proved to be an effective method in many instances; ${ }^{17}$

2. Elective coagulation of vascular connections; ${ }^{11}$ this is the logical and most consistent form of treatment.

\section{INTRAUTERINE DEATH}

The ante-partal death of one or several offspring of a multiple gestation occurs quite frequently (in about 1 to $5 \%$ of all multiple pregnancies).${ }^{14}$ In addition to the emotional and psychological strain this places on the parents, the state of the surviving offspring, whether one or several, must be closely monitored. ${ }^{12}$

When one of the monochorial offspring of a multiple gestation dies in utero, it is likely that the surviving offspring, whether one or several, will be subject to a high degree of neurological damage. The causes are generally seen in the embolization of thromboplastic material of the dead offspring into the surviving one.

\section{REDUCTION OF THE RISK OF PREMATURE DELIVERY}

The fact that multiples are subject to greater risks in their perinatal life than singleton children are has its causes in the high rate of premature births and the higher frequency of in utero maldevelopment of such children. ${ }^{10}$ The rate of complications for immature and underweight fetuses is around $40 \%$ for twins. The frequency of premature delivery is cited as $30 \%$ for twins and is thus higher by a factor of three to five than for comparable collectives of singleton pregnancies. Recognized preventive measures include the early diagnosis of multiple pregnancies, the transvaginal sonographic measurement of cervical length, ${ }^{9}$ an early certification by the physician that the mother is unfit for work (around 20 weeks of the pregnancy) and simply rest. Modern treatment methods for mothers carrying multiples who are not subject to any further risks no longer include recommendations for their inpatient treatment, for preventive cerclage or prophylactic tocolysis.

\section{INTRAUTERINE MALDEVELOPMENT}

Various factors contribute to intrauterine maldevelopment in multiple pregnancies: the mother's nutritional state, reduced uterine blood flow, anomalies of the umbilical chord, placental nutrient transport capacity, position of the placenta, unequal shares of the total placenta mass for the multiples and TTS. The frequency of such occurrences is cited as around $60 \%$ for offspring of multiple gestations. ${ }^{16}$

\section{INDUCTION OF LABOR}

In order to prevent the intrauterine death of the multiples near the term of the pregnancy, it is frequently recommended to induce labor following the completion of the 38th pregnancy week. Should a vaginal birth be intended, generally cervix maturation is obtained using prostaglandins. A cesarean section is performed when the following indications are given at this pregnancy stage and is in fact the primary method:

- Triplets or higher-order multiples;

- Position of the first multiple in a pelvic presentation or oblique presentation;

- Birth weight predicted by ultrasound examination of the second twin greater than that of the first twin by more than 500 gm;

- Birth weight predicted by ultrasound examination of twins lower than $1,800 \mathrm{gm}$;

- Monoamniotic twins (section at pregnancy week 34+0).

\section{REFERENCES}

1. Adegbite AL, Castille S, Ward S, Bajoria R. Neuromorbidity in preterm twins in relation to chorionicity and discordant birth weight. Am J Obstet Gynecol 2004;190:156.

2. Anderson RL, Goldberg JD, Goldbus MS. Prenatal diagnosis in multiple gestation: 20 years experience with amniocentesis. Prenat Diagn 1991;11:263.

3. Berkowitz RL, Lynch L, Lapinski R, Berger P. First trimester transabdominal multifetal pregnancy reduction: A report of two hundred completed cases. Am J Obstet Gynecol 1993;169:17. 
4. Berry SM, Puder KS, Battoms SF, Uckele JE, Romero R, Cotton DB. Comparison of intrauterine hematologic and biochemical values between twin pairs with and without stuck twin syndrome. Am J Obstet Gynecol 1995;172:1403.

5. Blickstein I. The twin-twin transfusion syndrome. Obstet Gynecol 1990;76:714.

6. Boklage CE. Survival probability of human conceptions from fertilization to term. Int J Fertil 1990;35.

7. Eriksson AW, Bressers WMA, Kostense PJ, et al. Twinning rate in Scandinavia, Germany and the Netherlands during years of privation. Acta Gen Med Genell 1988;37:277.

8. Evans MI, Goldberg JD, Dommergues M, Wagner RJ. Efficacy of second-trimester selective termination for fetal abnormalities: International collaborative experience among the world's largest centers. Am J Obstet Gynecol 1994;171:9.

9. Fuchs I, Tsoi E, Henrich W, Dudenhausen JW, Nicolaides KH. Sonographic measurement of cervical length in twin pregnancies in threatened preterm labor. Ultrasound Obstet Gynecol 2004;23:42.

10. Garite TJ, Clark RH, Elliott JP, Thorp JA. Twins and triplets: The effect of plurality and growth on neonatal outcome compared with singleton infants. Am J Obstet Gynecol 2004;191:700.

11. Hecher K, Hackeiöer BJ, Vitle Y. Umbilical cord coagulation by microendoscopy at 16 weeks gestation in a cardiac twin. Ultrasound Obstet Gynecol 1997;10:130.
12. Kilby MD, Govind A, O’Brien PM. Outcome of twin pregnancies complicated by a single intrauterine death: A comparison with viable twin pregnancies. Obstet Gynecol 1994;84:107.

13. Lee YM, Clearly-Goldman J, Thaker HM, Simpson LL. Antenatal sonographic prediction of twin chorionicity. Am J Obstet Gynecol 2006;195:863.

14. Lee YM, Wylie BJ, Simpson LL, D’Alton ME. Twin chorionicity and the risk of stillbirth. Obste Gynecol 2008;111:301.

15. Machin GA. Why is it important to diagnose chorionicity and how do we do it? Best Pract Res Clin Obste Gyaenecol 2004;18:515.

16. Maier RF, Bialobrzeski B, Gross A, Vogel M, Dudenhausen JW, Obladen M. Acute and chronic fetal hypoxia in monochorionic and dichorionic twins. Obstet Gynecol 1995;86:973.

17. Saunders NJ, Snijders RJ, Nicolaides KH. Therapeutic amniocentesis in twin-twin transfusion syndrome appearing in the second trimester of pregnancy. Am J Obstet Gynecol 1992;166:820.

18. Sebire NJ, Souka A, Skentou H, Geerts L, Nicolaides KH. Early prediction of severe twin-to-twin transfusion syndrome. Hum Reprod 2000;15:2008. 